\title{
A Korovkin-type approximation theorem for double sequences of positive linear operators of two variables in statistical $A$-summability sense
}

Sevda Orhan, Fadime Dirik, and Kamil Demirci 


\title{
A KOROVKIN-TYPE APPROXIMATION THEOREM FOR DOUBLE SEQUENCES OF POSITIVE LINEAR OPERATORS OF TWO VARIABLES IN STATISTICAL $A$-SUMMABILITY SENSE
}

\author{
SEVDA ORHAN, FADIME DIRIK, AND KAMIL DEMIRCI
}

Received 30 November, 2012

\begin{abstract}
In this paper, using the concept of statistical $A$-summability which is stronger than classical convergence and $A$-statistical convergence, we obtain a Korovkin-type approximation theorem for double sequences of positive linear operators of two variables from $H_{w}(K)$ to $C_{B}(K)$. Also, we give an example such that our new approximation result works but its classical and $A$-statistical cases do not work.
\end{abstract}

2010 Mathematics Subject Classification: 41A25; 41A36;47B38

Keywords: statistical $A$-summability for double sequences, positive linear operator, Korovkin type approximation theorem, the Bleimann, Butzer and Hahn operators

\section{INTRODUCTION}

The study of the Korovkin-type approximation theory is an area of active research, which deals with the problem of approximating a function by means of a sequence of positive linear operators. Later many researchers investigated approach conditions for various operators defined on different spaces. Using the concept of statistical convergence in approximation theory provides us with many advantages. In particular, the matrix summability methods of Cesáro type are strong enough to correct the lack of convergence of various sequences of linear operators such as the interpolation operator of Hermite-Fejér [15], because these types of operators do not converge at points of simple discontinuity. Furthermore, in recent years, with the help of the concept of uniform statistical convergence, which is a regular (non-matrix) summability transformation, various statistical approximation results have been proved $[1-3,5,6,9,14]$. Then, it was demonstrated that those results are more powerful than the classical Korovkin theorem. Also, Erkus and Duman [8] have studied a Korovkin type approximation theorem via $A$-statistical convergence in the space $H_{\omega}(K)$ (was given in [10]) where $K=[0, \infty) \times[0, \infty)$. Later, using the concept of the equi-statistical convergence which is more powerful than classical convergence and statistical convergence, Karakuş and Demirci have obtained the Korovkin-type approximation theorem for positive linear operators defined on $H_{\omega}(K)$ in [13]. 
Our primary interest in the present paper is to obtain a general Korovkin type approximation theorem for double sequences of positive linear operators of two variables from $H_{\omega}(K)$ to $C_{B}(K)$ via statistical $A$-summability.

Let us first remind of the concept of $A$-statistical convergence for double sequences.

A double sequence $x=\left(x_{m, n}\right)$ is said to be convergent in Pringsheim's sense if, for every $\varepsilon>0$, there exists $N=N(\varepsilon) \in \mathbb{N}$, the set of all natural numbers, such that $\left|x_{m, n}-L\right|<\varepsilon$ whenever $m, n>N$, where $L$ is called the Pringsheim limit of $x$ and denoted by $P-\lim _{m, n} x_{m, n}=L$ (see [20]). We shall call such an $x$, briefly, " $P$ convergent". A double sequence is called bounded if there exists a positive number $M$ such that $\left|x_{m, n}\right| \leq M$ for all $(m, n) \in \mathbb{N}^{2}=\mathbb{N} \times \mathbb{N}$. Note that in contrast to the case for single sequences, a convergent double sequence need not to be bounded. Let $A=\left(a_{j, k, m, n}\right)$ be a four-dimensional summability matrix. For a given double sequence $x=\left(x_{m, n}\right)$, the $A$-transform of $x$, denoted by $A x:=\left((A x)_{j, k}\right)$, is given by

$$
(A x)_{j, k}=\sum_{(m, n) \in \mathbb{N}^{2}} a_{j, k, m, n} x_{m, n}
$$

provided the double series converges in Pringsheim's sense for every $(j, k) \in \mathbb{N}^{2}$.

A two dimensional matrix transformation is said to be regular if it maps every convergent sequence in to a convergent sequence with the same limit. The wellknown characterization for two dimensional matrix transformations which are regular is known as Silverman-Toeplitz conditions (see, for instance, [12]). In 1926, Robison [21] presented a four dimensional analog of the regularity by considering an additional assumption of boundedness. This assumption was made because a double $P$-convergent sequence is not necessarily bounded. The definition and the characterization of regularity for four dimensional matrices is known as Robison-Hamilton conditions, or briefly, $R H$-regularity (see $[11,21]$ ).

Recall that a four dimensional matrix $A=\left(a_{j, k, m, n}\right)$ is said to be $R H$-regular if it maps every bounded $P$-convergent sequence into a $P$-convergent sequence with the same $P$-limit. The Robinson-Hamilton conditions state that a four dimensional matrix $A=\left(a_{j, k, m, n}\right)$ is $R H$-regular if and only if

(i) $P-\lim _{j, k} a_{j, k, m, n}=0$ for each $(m, n) \in \mathbb{N}^{2}$,

(ii ) $P-\lim _{j, k} \sum_{(m, n) \in \mathbb{N}^{2}} a_{j, k, m, n}=1$,

(iii) $P-\lim _{j, k} \sum_{m \in \mathbb{N}}\left|a_{j, k, m, n}\right|=0$ for each $n \in \mathbb{N}$,

(iv) $P-\lim _{j, k} \sum_{n \in \mathbb{N}}\left|a_{j, k, m, n}\right|=0$ for each $m \in \mathbb{N}$, 
(v) $\sum_{(m, n) \in \mathbb{N}^{2}}\left|a_{j, k, m, n}\right|$ is $P$-convergent for every $(j, k) \in \mathbb{N}^{2}$,

(vi) there exist finite positive integers $A$ and $B$ such that $\sum_{m, n>B}\left|a_{j, k, m, n}\right|<A$ holds for every $(j, k) \in \mathbb{N}^{2}$.

Now let $A=\left(a_{j, k, m, n}\right)$ be a non-negative $R H$-regular summability matrix, and let $K \subset \mathbb{N}^{2}$. Then the $A$-density of $K$ is given by

$$
\delta_{2}^{A}\{K\}:=P-\lim _{j, k} \sum_{(m, n) \in K(\varepsilon)} a_{j, k, m, n}
$$

where

$$
K(\varepsilon):=\left\{(m, n) \in \mathbb{N}^{2}:\left|x_{m, n}-L\right| \geq \varepsilon\right\}
$$

provided that the limit on the right-hand side exists in Pringsheim's sense. A real double sequence $x=\left(x_{m, n}\right)$ is said to be $A$-statistically convergent to a number $L$ if, for every $\varepsilon>0$,

$$
\delta_{2}^{A}\left\{(m, n) \in \mathbb{N}^{2}:\left|x_{m, n}-L\right| \geq \varepsilon\right\}=0 .
$$

In this case, we write $s t_{2}^{A}-\lim _{m, n} x=L$. Clearly, a $P$-convergent double sequence is $A$-statistically convergent to the same value but its converse is not always true. Also, note that an $A$-statistically convergent double sequence need not to be bounded. For example, consider the double sequence $x=\left(x_{m, n}\right)$ given by

$$
x_{m, n}= \begin{cases}1, & \text { if } m \text { and } n \text { are squares, } \\ 0, & \text { otherwise. }\end{cases}
$$

We should note that if we take $A=C(1,1)$, which is the double Cesáro matrix, then $C(1,1)$-statistical convergence coincides with the notion of statistical convergence for double sequence, which was introduced in [17,19]:

If $E \subset \mathbb{N}^{2}$ is a two-dimensional subset of positive integers, then $E_{j, k}$ denotes the set $(m, n) \in E: m \leq j, n \leq k$ and $\left|E_{j, k}\right|$ denotes the cardinality of $E_{j, k}$. The double natural density of $E[17,19]$ is given by

$$
\delta_{2}(E):=P-\lim _{j, k} \frac{1}{j k}\left|E_{j, k}\right|,
$$

if it exists. The number sequence $x=\left(x_{m, n}\right)$ is statistically convergent to $L$ provided that for every $\varepsilon>0$, the set $E:=E(\varepsilon):=\left\{m \leq j, n \leq k:\left|x_{m, n}-L\right| \geq \varepsilon\right\}$ has natural density zero; i.e.,

$$
P-\lim _{j, k} \frac{1}{j k}\left|\left\{m \leq j, n \leq k:\left|x_{m, n}-L\right| \geq \varepsilon\right\}\right|=0,
$$

in that case we write $s t_{2}-\lim _{m, n} x_{m, n}=L$. 
Finally, if we replace the matrix $A$ by the identity matrix for four dimensional matrices, then $A$-statistical convergence reduces to the Pringsheim convergence.

Recently, the idea of statistical $(C, 1)$-summability was introduced in [16] and of statistical $(H, 1)$-summability in [18] by Moricz. Then these statistical summability methods were generalized by defining the statistical $A$-summability in [7]. The concept of statistical $A$-summability for double sequences has defined by Karakuş and Demirci [4].

Hence, we know that if a double sequence is bounded and $A$-statistical convergent to $L$, then it is $A$-summable to $L$, and hence statistically $A$-summable to $L$ but not conversely [4].

Now we recall the following definition for a double sequence.

Definition 1 ([4]). Let $A=\left(a_{j, k, m, n}\right)$ be a non-negative $R H$-regular summability matrix and $x=\left(x_{m, n}\right)$ be a double sequence. We say that $x$ is statistically $A$-summable to $L$ if for every $\varepsilon>0$,

$$
\delta_{2}\left\{(j, k) \in \mathbb{N}^{2}:\left|(A x)_{j, k}-L\right| \geq \varepsilon\right\}=0,
$$

i.e.,

$$
P-\lim _{m, n} \frac{1}{m n}\left|\left\{j \leq m, k \leq n:\left|(A x)_{j, k}-L\right| \geq \varepsilon\right\}\right|=0,
$$

where $(A x)_{j, k}$ is as in (1.1).

Thus, the double sequence $x$ is statistically $A$-summable to $L$ if and only if $A x:=$ $\left((A x)_{j, k}\right)$ is statistically convergent to $L$.

\section{A KOROVKIN-TYPE APPROXIMATION THEOREM}

We denote by $C_{B}(K)$ the space of all continuous and bounded real valued functions on $K=[0, \infty) \times[0, \infty)$. This space is equipped with the supremum norm

$$
\|f\|=\sup _{(x, y) \in K}|f(x, y)|,\left(f \in C_{B}(K)\right) .
$$

Consider the space $H_{\omega}(K)$ of all real valued functions $f$ on $K$ and satisfying

$$
|f(u, v)-f(x, y)| \leq \omega\left(\left|\frac{u}{1+u}-\frac{x}{1+x}\right|,\left|\frac{v}{1+v}-\frac{y}{1+y}\right|\right)
$$

where $\omega$ be a function of the type of the modulus of continuity given by, for $\delta, \delta_{1}, \delta_{2}>$ 0 ,

(1) $\omega$ is non-negative increasing function on $K$ with respect to $\delta_{1}, \delta_{2}$,

(2) $\omega\left(\delta, \delta_{1}+\delta_{2}\right) \leq \omega\left(\delta, \delta_{1}\right)+\omega\left(\delta, \delta_{2}\right)$,

(3) $\omega\left(\delta_{1}+\delta_{2}, \delta\right) \leq \omega\left(\delta_{1}, \delta\right)+\omega\left(\delta_{2}, \delta\right)$,

(4) $\lim _{\delta_{1}, \delta_{2} \rightarrow 0} \omega\left(\delta_{1}, \delta_{2}\right)=0$. 
Then it is obvious, by (4) that any function in $H_{\omega}(K)$ is continuous on $K$. Also, any function $f \in H_{\omega}(K)$ satisfies the inequality

$$
|f(x, y)| \leq|f(0,0)|+\omega(1,1), \quad x, y \geq 0
$$

and therefore is bounded on $K$. So, $H_{\omega}(K) \subset C_{B}(K)$.

We also use the following test functions

$$
\begin{gathered}
f_{0}(u, v)=1, f_{1}(u, v)=\frac{u}{1+u}, f_{2}(u, v)=\frac{v}{1+v} \\
\text { and } f_{3}(u, v)=\left(\frac{u}{1+u}\right)^{2}+\left(\frac{v}{1+v}\right)^{2} .
\end{gathered}
$$

Now we have the following result.

Theorem 1. Let $\left\{L_{m, n}\right\}$ be a sequence of positive linear operators from $H_{\omega}(K)$ into $C_{B}(K)$ and let $A=\left(a_{j, k, m, n}\right)$ be a nonnegative $R H$-regular summability matrix. Assume that the following conditions hold:

$$
s t_{2}-\lim \left\|\sum_{(m, n)} a_{j, k, m, n} L_{m, n}\left(f_{i}\right)-f_{i}\right\|=0, i=0,1,2,3 .
$$

Then, for any $f \in H_{\omega}(K)$,

$$
s t_{2}-\lim \left\|\sum_{(m, n)} a_{j, k, m, n} L_{m, n}(f)-f\right\|=0 .
$$

Proof. Assume that (2.1) holds. Let $f \in H_{\omega}(K)$ and $(x, y) \in K$ be fixed. Since $f \in H_{\omega}(K)$, for all $(u, v) \in K$, we write

$$
|f(u, v)-f(x, y)| \leq \varepsilon+\frac{2 N}{\delta^{2}}\left\{\left(\frac{u}{1+u}-\frac{x}{1+x}\right)^{2}+\left(\frac{v}{1+v}-\frac{y}{1+y}\right)^{2}\right\}
$$

where $N:=\|f\|$. After some simple calculations, using the linearity and positivity of the operators $L_{m, n}$, we obtain

$$
\begin{aligned}
& \left|\sum_{(m, n)} a_{j, k, m, n} L_{m, n}(f ; x, y)-f(x, y)\right| \\
& \leq \varepsilon+C\left\{\left|\sum_{(m, n)} a_{j, k, m, n} L_{m, n}\left(f_{0} ; x, y\right)-f_{0}(x, y)\right|\right. \\
& \quad+\left|\sum_{(m, n)} a_{j, k, m, n} L_{m, n}\left(f_{1} ; x, y\right)-f_{1}(x, y)\right|
\end{aligned}
$$




$$
\begin{aligned}
& +\left|\sum_{(m, n)} a_{j, k, m, n} L_{m, n}\left(f_{2} ; x, y\right)-f_{2}(x, y)\right| \\
& \left.+\left|\sum_{(m, n)} a_{j, k, m, n} L_{m, n}\left(f_{3} ; x, y\right)-f_{3}(x, y)\right|\right\}
\end{aligned}
$$

where $C:=\max \left\{\varepsilon+N+\frac{2 N}{\delta^{2}}\left(\left(\frac{A}{1+A}\right)^{2}+\left(\frac{B}{1+B}\right)^{2}\right), \frac{4 N}{\delta^{2}} \frac{A}{1+A}, \frac{4 N}{\delta^{2}} \frac{B}{1+B}, \frac{2 N}{\delta^{2}}\right\}$.

Then, taking supremum over $(x, y) \in K$ we get

$$
\left\|\sum_{(m, n)} a_{j, k, m, n} L_{m, n}(f)-f\right\| \leq \varepsilon+C \sum_{i=0}^{3}\left\|\sum_{(m, n)} a_{j, k, m, n} L_{m, n}\left(f_{i}\right)-f_{i}\right\| .
$$

For a given $r>0$, choose $\varepsilon>0$ such that $\varepsilon<r$. Then, for each $i=0,1,2,3$, setting

$$
\begin{aligned}
U & :=\left\{(j, k):\left\|\sum_{(m, n)} a_{j, k, m, n} L_{m, n}(f)-f\right\| \geq r\right\}, \\
U_{i} & :=\left\{(j, k):\left\|\sum_{(m, n)} a_{j, k, m, n} L_{m, n}\left(f_{i}\right)-f_{i}\right\| \geq \frac{r-\varepsilon}{4 C}\right\},(i=0,1,2,3),
\end{aligned}
$$

it follows from (2.3) that

$$
U \subset \bigcup_{i=0}^{3} U_{i}
$$

which gives, for all $(j, k) \in \mathbb{N}^{2}$,

$$
\delta_{2}(U) \leq \sum_{i=0}^{3} \delta_{2}\left(U_{i}\right)
$$

From (2.1), we obtain (2.2). The proof is complete.

If we take $A=I$, which is the identity matrix for four dimensional matrices, in Theorem 1, we get the following statistical version of Theorem 1 .

Corollary 1. Let $\left\{L_{m, n}\right\}$ be a sequence of positive linear operators from $H_{\omega}(K)$ into $C_{B}(K)$. Assume that the following conditions hold:

$$
s t_{2}-\lim \left\|L_{m, n}\left(f_{i}\right)-f_{i}\right\|=0, i=0,1,2,3 .
$$

Then, for any $f \in H_{\omega}(K)$,

$$
s t_{2}-\lim \left\|L_{m, n}(f)-f\right\|=0 .
$$


In Corollary 1 , if the statistical convergence $(C(1,1)$-statistical convergence) replace with Pringsheim convergence, we obtain the following classical version of Theorem 1.

Corollary 2. Let $\left\{L_{m, n}\right\}$ be a sequence of positive linear operators from $H_{\omega}(K)$ into $C_{B}(K)$. Assume that the following conditions hold:

$$
P-\lim \left\|L_{m, n}\left(f_{i}\right)-f_{i}\right\|=0, i=0,1,2,3 .
$$

Then, for any $f \in H_{\omega}(K)$,

$$
P-\lim \left\|L_{m, n}(f)-f\right\|=0 .
$$

Remark 1. We now show that our result Theorem 1 is stronger than its classical version Corollary 2 and statistical version Corollary 1 . To see this first consider the following Bleimann, Butzer and Hahn operators of two variables:

$$
\begin{gathered}
T_{m, n}(f ; x, y)= \\
\frac{1}{(1+x)^{m}(1+y)^{n}} \sum_{k=0}^{m} \sum_{l=0}^{n} f\left(\frac{k}{m-k+1}, \frac{l}{n-l+1}\right)\left(\begin{array}{c}
m \\
k
\end{array}\right)\left(\begin{array}{l}
n \\
l
\end{array}\right) x^{k} y^{l},
\end{gathered}
$$

where $f \in H_{\omega}(K)$, and $K=[0, \infty) \times[0, \infty)$. It is known [8] that

$$
\begin{aligned}
T_{m, n}\left(f_{0} ; x, y\right)= & 1, \\
T_{m, n}\left(f_{1} ; x, y\right)= & \frac{m}{m+1} \frac{x}{1+x}, \\
T_{m, n}\left(f_{2} ; x, y\right)= & \frac{n}{n+1} \frac{y}{1+y}, \\
T_{m, n}\left(f_{3} ; x, y\right)= & \frac{m(m-1)}{(m+1)^{2}}\left(\frac{x}{1+x}\right)^{2}+\frac{m}{(m+1)^{2}} \frac{x}{1+x} \\
& +\frac{n(n-1)}{(n+1)^{2}}\left(\frac{y}{1+y}\right)^{2}+\frac{n}{(n+1)^{2}} \frac{y}{1+y} .
\end{aligned}
$$

Now take $A=C(1,1)$ and define a double sequence $u:=\left\{u_{m, n}\right\}$ by

$$
u_{m, n}=(-1)^{m+n}
$$

In this case, observe that

$$
s t_{2}-\lim C(1,1)(u)=0 .
$$

However, the double sequence $u$ is not $P$-convergent and statistical convergent. Now using (2.4) and (2.6), we define the following double positive linear operators on $H_{\omega}(K)$ as follows:

$$
L_{m, n}(f ; x, y)=\left(1+u_{m, n}\right) T_{m, n}(f ; x, y) .
$$


Then, observe that the double sequence of positive linear operators $\left\{L_{m, n}\right\}$ defined by (2.8) satisfy all hypotheses of Theorem 1 . Hence, by (2.5) and (2.7), we have, for all $f \in H_{\omega}(K)$,

$$
s t_{2}-\lim \left\|\sum_{(m, n)} a_{j, k, m, n} L_{m, n}(f)-f\right\|=0 .
$$

Since $u$ is not $P$-convergent and statistical convergent, the sequence $\left\{T_{m, n}(f)\right\}$ can not uniformly convergence to $f$ on $K$ or statistical sense.

\section{REFERENCES}

[1] G. A. Anastassiou and O. Duman, "A Baskakov type generalization of statistical Korovkin theory," J. Math. Anal. Appl., vol. 340, no. 1, pp. 476-486, 2008.

[2] G. A. Anastassiou and O. Duman, "Statistical fuzzy approximation by fuzzy positive linear operators," Comput. Math. Appl., vol. 55, no. 3, pp. 573-580, 2008.

[3] K. Demirci and F. Dirik, "A Korovkin type approximation theorem for double sequences of positive linear operators of two variables in A-statistical sense," Bull. Korean Math. Soc., vol. 47, no. 4, pp. 825-837, 2010.

[4] K. Demirci and S. Karakuş, "Korovkin-type approximation theorem for double sequences of positive linear operators via statistical $A$-summability," Result. Math., vol. 63, no. 1-2, pp. 1-13, 2013.

[5] O. Duman, M. K. Khan, and C. Orhan, " $A$-statistical convergence of approximating operators," Math. Inequal. Appl., vol. 6, no. 4, pp. 689-699, 2003.

[6] O. Duman, E. Erkuş, and V. Gupta, "Statistical rates on the multivariate approximation theory," Math. Comput. Modelling, vol. 44, no. 9-10, pp. 763-770, 2006.

[7] O. H. H. Edely and M. Mursaleen, "On statistical A-summability," Math. Comput. Modelling, vol. 49, no. 3-4, pp. 672-680, 2009.

[8] E. Erkuş and O. Duman, " $A$-statistical extension of the Korovkin type approximation theorem," Proc. Indian Acad. Sci., Math. Sci., vol. 115, no. 4, pp. 499-508, 2005.

[9] E. Erkuş, O. Duman, and H. M. Srivastava, "Statistical approximation of certain positive linear operators constructed by means of the Chan-Chyan-Srivastava polynomials," Appl. Math. Comput., vol. 182, no. 1, pp. 213-222, 2006.

[10] A. D. Gadjiev and O. Çakar, "On uniform approximation by Bleimann, Butzer and Hahn operators on all positive semiaxis," Trans. Acad. Sci. Azerb., Ser. Phys.-Tech. Math. Sci., vol. 19, no. 5, Math. Mech., pp. 21-26, 1999.

[11] H. J. Hamilton, “Transformations of multiple sequences," Duke Math. J., vol. 2, pp. 29-60, 1936.

[12] G. H. Hardy, Divergent series. Oxford: At the Clarendon Press (Geoffrey Cumberlege), 1949, vol. XIV.

[13] S. Karakuş and K. Demirci, "Equi-statistical extension of the Korovkin type approximation theorem,” Turk. J. Math., vol. 33, no. 2, pp. 159-168, 2009.

[14] S. Karakuş, K. Demirci, and O. Duman, "Equi-statistical convergence of positive linear operators," J. Math. Anal. Appl., vol. 339, no. 2, pp. 1065-1072, 2008.

[15] M. K. Khan and R. Bojanic, "Summability of Hermite-Fejér interpolation for functions of bounded variation," J. Nat. Sci. Math., vol. 32, no. 1, pp. 5-10, 1992.

[16] F. Móricz, "Tauberian conditions, under which statistical convergence follows from statistical summability $(C, 1)$, , J. Math. Anal. Appl., vol. 275, no. 1, pp. 277-287, 2002. 
[17] F. Móricz, "Statistical convergence of multiple sequences," Arch. Math., vol. 81, no. 1, pp. 82-89, 2003.

[18] F. Móricz, "Theorems relating to statistical harmonic summability and ordinary convergence of slowly decreasing or oscillating sequences," Analysis, München, vol. 24, no. 2, pp. 127-145, 2004.

[19] Mursaleen and O. H. H. Edely, "Statistical convergence of double sequences," J. Math. Anal. Appl., vol. 288, no. 1, pp. 223-231, 2003.

[20] A. Pringsheim, "Zur Theorie der zweifach unendlichen Zahlenfolgen," Math. Ann., vol. 53, pp. 289-321, 1900.

[21] G. M. Robison, "Divergent double sequences and series," Trans. Am. Math. Soc., vol. 28, pp. 50-73, 1926.

Authors' addresses

\section{Sevda Orhan}

Sinop University, Faculty of Sciences and Arts, Department of Mathematics, 57000 Sinop, Turkey

E-mail address: orhansevda@gmail.com

\section{Fadime Dirik}

Sinop University, Faculty of Sciences and Arts, Department of Mathematics, 57000 Sinop, Turkey E-mail address: dirikfadime@gmail.com

\section{Kamil Demirci}

Sinop University, Faculty of Sciences and Arts, Department of Mathematics, 57000 Sinop, Turkey

E-mail address: kamildesinop.edu.tr 